\title{
Putting ACCOMPLISH into context: management of hypertension in 2010
}

\author{
Finlay A. McAlister MD MSc, Robert J. Herman MD, Nadia A. Khan MD MSc, Simon W. Rabkin MD, \\ Norm Campbell MD; for the Canadian Hypertension Education Program
}

Previously published at www.cmaj.ca

$\mathrm{T}$ he much publicized Avoiding Cardiovascular events through COMbination therapy in Patients LIving with Systolic Hypertension (ACCOMPLISH) trial ${ }^{1}$ evaluated whether combination therapy with an angiotensinconverting enzyme (ACE) inhibitor (benazepril) and a dihydropyridine calcium channel blocker (amlodipine) was superior to combination therapy with the same ACE inhibitor and a thiazide diuretic (hydrochlorothiazide). Despite a statistically significant result favouring the combination of ACE inhibitor and calcium channel blocker, the clinical implication of this study remains a topic of substantial debate. Some hypertension experts have advocated changing national guidelines on the basis of these results, ${ }^{2}$ but others have been more circumspect. ${ }^{3}$ Our purpose here is to review the implications of ACCOMPLISH for Canadian clinicians.

ACCOMPLISH enrolled 11506 individuals over 55 years of age who had hypertension and were at high cardiovascular risk (see Appendix 1, available at www.cmaj.ca/cgi/content/full /cmaj.092142/DC1). Although 75\% of the participants were taking two or more antihypertensive agents before enrollment (and $38 \%$ were taking three drugs), blood pressure was uncontrolled (> 140/90 mm Hg) in two-thirds of participants. Patients in both study arms were started on combination therapy and underwent aggressive monthly up-titration to achieve target blood pressure. As a result, systolic blood pressure declined by about $14 \mathrm{~mm} \mathrm{Hg}$ in both treatment arms. ACCOMPLISH was stopped early because the primary outcome - a composite of cardiovascular events and death - was significantly lower in the group receiving the ACE inhibitor and calcium channel blocker $(9.6 \% \mathrm{v}$. $11.8 \%$ after three years, hazard ratio $0.80,95 \%$ confidence interval [CI] 0.72-0.90). Two-thirds of the primary end points consisted of admissions to hospital for unstable angina or coronary revascularization procedures, which has led some to argue that ACCOMPLISH merely demonstrated that amlodipine reduces anginal symptoms more effectively than a thiazide. Nonetheless, it is worth noting that there was a statistically significant benefit with the combination of ACE inhibitor and calcium channel blocker in terms of "death, nonfatal MI [myocardial infarction], or nonfatal stroke" (5.0\% v. 6.3\%, hazard ratio $0.79,95 \% \mathrm{CI}$ $0.67-0.92)$. In addition, the prespecified secondary renal outcome of "doubling of serum creatinine or development of endstage renal disease" was significantly less common among patients treated with ACE inhibitor and calcium channel blocker ( $2.0 \%$ v. $3.7 \%$, hazard ratio $0.52,95 \%$ CI $0.41-0.65){ }^{4}$

Was ACCOMPLISH a well-conducted active-control

\section{Key points}

- Most individuals with hypertension need more than one drug to achieve blood pressure targets.

- The low rates of cardiovascular events in both arms of the ACCOMPLISH trial relative to previous antihypertensive trials underscores the importance of prompt control of blood pressure.

- The ACCOMPLISH trial confirmed the benefits of combination therapy for high-risk individuals whose blood pressure is substantially above target.

trial $?^{5}$ In a word, yes. The ADVANCE Trial ${ }^{6}$ (Appendix 1, available at www.cmaj.ca/cgi/content/full/cmaj.092142/DC1) had previously proven that a combination of ACE inhibitor and thiazide, akin to the active comparator in ACCOMPLISH, was more efficacious than placebo for a similar patient population. The only methodologic concern with ACCOMPLISH is the fact that it was stopped early. Although this is not necessarily a problem, trials that are stopped early may overestimate treatment effects. ${ }^{7}$ This may be particularly relevant to interpreting the reported differences in renal outcomes in ACCOMPLISH. Although fewer patients treated with ACE inhibitor and calcium channel blocker experienced doubling of serum creatinine, ACCOMPLISH was stopped about one year earlier than usual for trials reporting renal outcomes. As such, the initial hemodynamic changes caused by each drug (amlodipine inducing a short-term rise and thiazide diuretics an early drop in glomerular filtration rate) had greater impact on an end point such as measured creatinine than would have been the case if follow-up had not been truncated. Indeed, the rates of decline in glomerular filtration rate after the first three months appeared similar in the two arms of ACCOMPLISH. ${ }^{8}$

The most striking result in ACCOMPLISH was the low rate of cardiovascular events in both arms relative to prior antihypertensive trials. For example, the Antihypertensive and Lipid-

From the Division of General Internal Medicine and Mazankowski Alberta Heart Institute (McAlister), University of Alberta, Edmonton, Alta.; the Departments of Medicine, of Community Health Sciences and of Physiology and Pharmacology (Herman, Campbell), Libin Cardiovascular Institute, University of Calgary, Calgary Alta.; and the Divisions of General Internal Medicine (Khan) and of Cardiology (Rabkin), University of British Columbia, Vancouver, BC

CMAJ 2010. DOI:10.1503/cmaj.092142

All editorial matter in CMAJ represents the opinions of the authors and not necessarily those of the Canadian Medical Association. 
Lowering treatment to prevent Heart Attack Trial (ALLHAT) ${ }^{9}$ enrolled patients of similar age and blood pressure. However, despite lower rates of cardiovascular disease and diabetes mellitus, the event rates in ALLHAT were nearly double those in ACCOMPLISH (Appendix 1, available at www.cmaj.ca/cgi /content/full/cmaj.092142/DC1). This observation emphasizes the value of achieving control of blood pressure quickly. Although $73 \%$ of the ACCOMPLISH participants achieved treatment goals within six months, only 55\% of those in ALLHAT met their targets by 12 months, and average systolic blood pressure at one year into the trial was nearly $6 \mathrm{~mm} \mathrm{Hg}$ higher in ALLHAT than in ACCOMPLISH. Indeed, the rapidity of achieving blood pressure treatment goals has been correlated with long-term prognosis in other hypertension trials, even if differences in blood pressure between patients with immediate and late response disappeared after only a few months. ${ }^{10}$ Therein lies the problem for blood pressure treatment based on stepped care, whereby drugs are initiated sequentially with observation for response after each step: most individuals with hypertension need more than one drug to achieve targets. Starting with two drugs may enable the clinician to achieve control of blood pressure more rapidly, but whether the use of fixed-dose combinations improves tolerability, adherence with therapy and costeffectiveness are unanswered questions that are still under study.

Before deciding on the implications of ACCOMPLISH for Canadian clinicians, three other facts are worthy of comment. First, ACCOMPLISH was not a trial of patients with newly diagnosed, uncomplicated hypertension; rather, it enrolled older individuals at high risk for cardiovascular events in whom initial therapy had already failed. Second, ACCOMPLISH reported a significant difference in clinical outcomes between treatment arms despite minimal differences in blood pressure control. This result contrasts with prior evidence that the most important predictor of outcomes with antihypertensive therapy is the extent to which blood pressure is reduced. ${ }^{11}$ It is possible that certain drug combinations may exert different effects and that pharmacogenomics may help to guide the choice of drug combinations in the future, but these hypotheses must be tested. The medical literature provides numerous examples of initially impressive treatment effects that have been found to be smaller (or that have been disproved) in subsequent studies. ${ }^{12}$ Therefore, caution is warranted in extrapolating from the first study reporting an unexpected finding. Although the various antihypertensive classes endorsed in the guidelines of the Canadian Hypertension Education Program (ACE inhibitors, angiotensin receptor blockers, calcium channel blockers, thiazides and $\beta$-blockers for younger patients) appear equivalent when used as first-line monotherapy, ${ }^{11}$ testing different combinations head-to-head is the next frontier in blood pressure trials. Finally, it is important to emphasize that most strokes and myocardial infarctions occur in individuals with systolic blood pressure in the range of $130-150 \mathrm{~mm} \mathrm{Hg} .{ }^{13}$

What are the implications of ACCOMPLISH? In our opinion, ACCOMPLISH confirms the benefit of combination therapy for high-risk individuals with hypertension and thus supports the recommendation of the Canadian Hypertension Education Program to start combination therapy for patients with systolic blood pressure that is more than $20 \mathrm{~mm} \mathrm{Hg}$ above target (www.hypertension.ca). Although a combination of ACE inhibitor and calcium channel blocker is appropriate for highrisk patients similar to those enrolled in ACCOMPLISH, clinicians should individualize the choice of which drugs to combine according to patients' particular combinations of comorbidities.

\section{This article has been peer reviewed.}

Competing interests: At the time this article was prepared, all authors were members of the Canadian Hypertension Education Program. Between 2006 and 2009, Norm Campbell sat on advisory boards for Aventis, Biovail, Boehringer-Ingelhiem, Novartis, Pfizer, Servier and Schering Plough; was a paid consultant for Novartis and Solvay; received speaking honoraria from Bayer, Biovail, BMS/Sanofi, Boehringer-Ingelhiem, Merck, Novartis, Pfizer and Servier; and received travel support from Bayer, Biovail, BMS, Schering Plough and Servier. Simon Rabkin served as a consultant to Bayer and has received honoraria for speaking at continuing medical education events from Abbott, Nycomed, Schering Plough and Servier. None declared for Finlay McAlister, Robert Herman and Nadia Khan.

Contributors: Finlay McAlister conceived the idea for this commentary. All authors contributed to the writing and revision of the article for important intellectual content, and all approved the final version to be published.

Acknowledgements: Finlay McAlister is supported by an Alberta Heritage Foundation for Medical Research Senior Health Scholar Award and holds the University of Alberta/Merck Frosst/Aventis Chair in Patient Health Management. Norm Campbell holds the Canada Chair in Hypertension Prevention and Control funded by the Canadian Institutes of Health Research, the Canadian Hypertension Society and Sanofi Aventis. Nadia Khan receives salary support from a CIHR New Investigator award.

\section{REFERENCES}

1. Jamerson K, Weber MA, Bakris GL, et al.; ACCOMPLISH Trial Investigators. Benazepril plus amlodipine or hydrochlorthiazide for hypertension in high-risk patients. N Engl J Med 2008;359:2417-28.

2. Zanchetti A, Mancia G, Black HR, et al. Facts and fallacies of blood pressure control in recent trials: implications in the management of patients with hypertension. J Hypertens 2009;27:673-9.

3. Chobanian AV. Does it matter how hypertension is controlled? N Engl J Med 2008; 359:2485-8.

4. Bakris GL, Sarafidis PA, Weir MR, et al. Renal outcomes with different fixed-dose combination therapies in patients with hypertension at high risk for cardiovascular events (ACCOMPLISH): a prespecified secondary analysis of a randomised controlled trial. Lancet 2010;375:1173-81.

5. McAlister FA, Sackett DL. Active-control equivalence trials and antihypertensive agents. Am J Med 2001;111:553-8.

6. ADVANCE Collaborative Group. Effects of a fixed combination of perindopril and indapamide on macrovascular and microvascular outcomes in patients with type 2 diabetes mellitus (the ADVANCE trial): a randomized controlled trial. Lancet 2007:370:829-40.

7. Montori VM, Devereaux PJ, Adhikari NK, et al. Randomized trials stopped early for benefit: a systematic review. JAMA 2005;294:2203-9.

8. Heerspink HL, de Zeeuw D. Composite renal endpoints: Was ACCOMPLISH accomplished? Lancet 2010;375:1140-2.

9. ALLHAT Collaborative Research Group. Major outcomes in high-risk hypertensive patients randomized to angiotensin-converting enzyme inhibitor or calcium channel blocker vs diuretic. The Antihypertensive and Lipid-Lowering Treatment to Prevent Heart Attack Trial (ALLHAT). JAMA 2002;288:2981-97.

10. Weber MA, Julius W, Kjeldsen SE, et al. Blood pressure dependent and independent effects of antihypertensive treatment on clinical events in the VALUE Trial. Lancet 2004;363:2049-51.

11. Law MR, Morris JK, Wald NJ. Use of blood pressure lowering drugs in the prevention of cardiovascular disease: meta-analysis of 147 randomised trials in the contex of expectations from prospective epidemiological studies. BMJ 2009;338:b1665. doi: $10.1136 /$ bmj.b1665.

12. Ioannidis JP. Contradicted and initially stronger effects in highly cited clinical research. JAMA 2005;294:218-28.

13. Lawes CMM, Vander Hoorn S, Law MR, et al. Blood pressure and the global burden of disease 2000. Part II: Estimates of attributable burden. J Hypertens 2006;24: 423-30.

Correspondence to: Dr. Finlay A. McAlister, 2F1.21 WMC,

University of Alberta Hospital, 8440112 St., Edmonton AB

T6G 2R7; Finlay.McAlister@ualberta.ca 